Supplement of Biogeosciences, 17, 2041-2059, 2020

https://doi.org/10.5194/bg-17-2041-2020-supplement

(C) Author(s) 2020. This work is distributed under

the Creative Commons Attribution 4.0 License.

(c) (1)

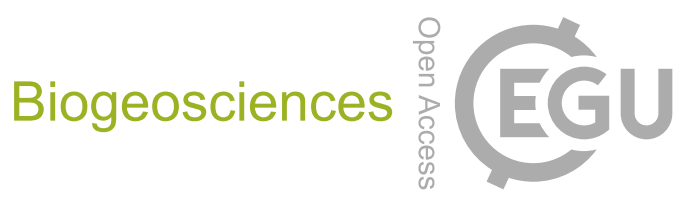

Supplement of

\title{
Drivers and modelling of blue carbon stock variability in sediments of southeastern Australia
}

Carolyn J. Ewers Lewis et al.

Correspondence to: Carolyn J. Ewers Lewis (ce8dp@virginia.edu)

The copyright of individual parts of the supplement might differ from the CC BY 4.0 License. 
Table S1. Hypothesized drivers of 30-cm deep sediment blue C stock variability. Potential drivers were identified in the literature, as described in the text and under "Hypothesis and rationale" of this table. These drivers were grouped into three categories: 1) ecological (ecosystem type and dominant species/ecological vegetation class), 2) geomorphological (elevation, slope, distance to freshwater channel, distance to coast, and lithology), and 3) anthropogenic (land use and population). Sources and resolution (where appropriate) for spatial data used as driver proxies in our modelling are described in the far right column.

\begin{tabular}{|c|c|c|}
\hline Driver & Hypothesis and rationale & Spatial data source and resolution \\
\hline \multicolumn{3}{|l|}{ Ecological } \\
\hline $\begin{array}{l}\text { ECOSYSTEM } \\
\text { TYPE } \\
\text { Tidal marsh, } \\
\text { mangrove, or } \\
\text { seagrass }\end{array}$ & $\begin{array}{l}\text { Ecosystem is the dominant driver of C stock variability } \\
\text { C stocks of tidal marshes }>\text { mangrove forests > seagrass meadows (Ewers Lewis et al., 2018; } \\
\text { Siikamäki et al., 2013). } \\
\text { C accumulation is driven by position in the tidal frame, therefore differs by ecosystem because } \\
\text { each occupy a different inundation range in tidal frame (Saintilan et al., 2013). } \\
\text { Differences in above- and below-ground plant biomass influence C stocks. Root C may be } \\
\text { particularly important, as sediment C at depth in southeast Australian saltmarsh and mangrove has } \\
\text { been observed to be dominated by root C and relatively low in allochthonous C, including leaf } \\
\text { litter (Saintilan et al., 2013). } \\
\text { Morphology impacts wave energy and particle settling (Mudd et al., 2010), as well as direct } \\
\text { trapping by vegetation to the sediment surface (Chen et al., 2018). }\end{array}$ & $\begin{array}{l}\text { Saltmarsh \& mangrove extent: Boon } \\
\text { et al., } 2011 \\
\text { Seagrass extent: Blake et al., 2000; } \\
\text { Blake and Ball, 2001b, 2001a; Roob } \\
\text { et al., 1998; Roob and Ball, } 1997\end{array}$ \\
\hline $\begin{array}{l}\text { DOMINANT } \\
\text { SPECIES/EVC } \\
\text { Dominant species } \\
\text { (seagrass, mangrove); } \\
\text { Ecological } \\
\text { Vegetation Class } \\
\text { (tidal marshes) }\end{array}$ & $\begin{array}{l}\text { Species composition better explains C stock variability than ecosystem alone } \\
\text { C stocks vary across species (in seagrasses, e.g. Jankowska et al., 2016; Lavery et al., 2013; in } \\
\text { tidal marsh plants, e.g. Sousa et al., 2010) and community composition (e.g. species richness in } \\
\text { mangroves, Atwood et al. 2017). } \\
\text { Species within a community occur at different elevations and positions in the tidal frame, which } \\
\text { can impact sedimentation and C capture (e.g. Kelleway et al. 2017). }\end{array}$ & $\begin{array}{l}\text { Saltmarsh EVC \& mangrove extent: } \\
\text { Boon et al., } 2011 \\
\text { Seagrass extent: (Blake et al., 2000; } \\
\text { Blake and Ball, 2001b, 2001a; Roob } \\
\text { et al., 1998; Roob and Ball, 1997) }\end{array}$ \\
\hline \multicolumn{3}{|l|}{ Geomorphological } \\
\hline ELEVATION & $\begin{array}{l}\text { Lower elevations are correlated with higher C stocks } \\
\text { Lower elevations have higher sedimentation rates, aiding the trapping of organic C from } \\
\text { organisms growing on soil surfaces (Connor et al., 2001). } \\
\text { Lower elevations are inundated more often providing more opportunity for contribution of } \\
\text { allochthonous C via particles and C settling out of the water column (Chen et al., 2015; Chmura et } \\
\text { al., 2003; Chmura and Hung, 2004). }\end{array}$ & $\begin{array}{l}\text { Victorian Coastal Digital Elevation } \\
\text { Model (VCDEM 2017) } \\
2.5 \mathrm{~m} \text { and } 10 \mathrm{~m} \text { resolutions } \\
\text { Cooperative Research Centre for } \\
\text { Spatial Information (CRCSI) }\end{array}$ \\
\hline SLOPE & $\begin{array}{l}\text { Shallower slopes are correlated with higher } \mathrm{C} \text { stocks } \\
\text { Steeper slopes are more vulnerable to erosion and less conducive to sedimentation and particle } \\
\text { trapping than shallower slopes. }\end{array}$ & $\begin{array}{l}\text { Calculated in ArcMap from } 2.5 \mathrm{~m} \\
\text { and } 10 \mathrm{~m} \text { elevation data (above) }\end{array}$ \\
\hline
\end{tabular}




\section{DISTANCE TO FRESHWATER}

CHANNEL

Euclidean distance to

closest channe
Distance to freshwater channel is negatively correlated with C stocks

- Sediment accretion rates (positively correlated to organic matter) decrease with distance from freshwater channels as elevation increases and inundation is less frequent (Chmura and Hung, 2004).

$>$ Higher freshwater inputs may lead to higher production of autochthonous C (Kelleway et al., 2016).

$>$ Delivery of nutrients from terrestrial sources may increase plant growth (Armitage and Fourqurean, 2016)

$>$ Fluvial environments are associated with smaller grain size particles, which enhance C preservation compared to sandy substrates (Kelleway et al., 2016; Saintilan et al., 2013)

\section{DISTANCE TO}

\section{COAST}

Euclidean distance to

closest point on the coast

\section{LITHOLOGY} the greatest proportion of the catchment
Rock type that covers

$\mathrm{C}$ stocks are greater higher up in the catchment

$>\mathrm{C}$ stocks in fluvial environments $>$ marine environments (e.g. in tidal marshes, Van De Broek et al., 2016; Kelleway et al., 2016; Macreadie et al., 2017a; Saintilan et al., 2013).

Fluvially located tidal marshes have on average over $2 \mathrm{x}$ higher $\mathrm{C}$ stocks compared to marine tidal marshes (Kelleway et al., 2017; Macreadie et al., 2017a).

Greater inputs of suspended particulate organic $\mathrm{C}$ from terrestrial sources higher in the catchment (Van De Broek et al., 2016).

Amount of deeper, stable $\mathrm{C}$ in tidal marshes decreased from the upper estuary toward the coast (Van De Broek et al., 2016).

C stocks vary with terrestrial parent material of sediments

Terrestrial weathering, erosion, and sediment properties influence organic C export (Galy et al. 2007)

$>$ Rock type may influence grain size of sediments exported from catchments; smaller grain sizes enhance C preservation (Kelleway et al., 2016; Saintilan et al., 2013), bind more organic molecules relative to coarse particles (Mayer, 1994), and can be associated with high C stocks (e.g. Serrano et al. 2016)

Mineral content affects organic C quantity, preservation, and flux (Torn et al., 1997).

\section{Anthropogenic}

\section{LAND USE}

Represented as

proportion of

catchment area that

1) urbanized

2) agricultural

3) natural primary land use is
C stocks vary based on land use activities in the catchment

> Land use may impact export of terrestrial $\mathrm{C}$ contributing to allochthonous $\mathrm{C}$ inputs to blue $\mathrm{C}$ ecosystems; allochthonous $\mathrm{C}$ can contribute up to $50 \%$ of $\mathrm{C}$ stocks (e.g. in seagrasses, Kennedy et al. 2010)

$>$ Nutrient inputs (e.g. from sewage effluent and agricultural runoff) can increase productivity, but have a negative impact on net $\mathrm{C}$ stocks (Armitage and Fourqurean, 2016; Kearns et al., 2018; Macreadie et al., 2017b)

Erosion of fine sediments in urbanized and agricultural regions may enhance sedimentation and C

burial (Mazarrasa et al., 2017; Serrano et al., 2016b; Yang et al., 2003).
Vicmap Hydro

$1: 25,000$

Department of Environment, Land,

Water \& Planning

Victoria State Government
Victorian Coastline 2008

Department of Environment, Land,

Water \& Planning

Victoria State Government

\section{1:100,000}

Geomorphology of Victoria

Department of Economic

Development, Jobs, Transport and

Resources

Victoria State Government

Victorian Land Use Information

System 2014/2015

Department of Economic

Development, Jobs, Transport and

Resources

Victoria State Government 
POPULATION

Mean population

C stocks differ across population levels due to a correlation with land use

Increases in population size lead to increases in urbanisation and competition for land use.
Population Density, Australia 2011

Australian Bureau of Statistics

density of the

catchment 
Table S2. Lithologies in Victoria

\begin{tabular}{|r|l|}
\hline Raster Code & Lithology \\
\hline 0 & other \\
\hline 1 & Aeolian \\
\hline 2 & Aeolian and Alluvium \\
\hline 3 & Alluvial \\
\hline 4 & Alluvium \\
\hline 5 & Basalt \\
\hline 6 & Colluvial \\
\hline 7 & Duricrust \\
\hline 8 & Fluvial \\
\hline 9 & Fluvial Aeolian \\
\hline 10 & Granite \\
\hline 11 & Granitic \\
\hline 12 & Lacustrine/Aeolian \\
\hline 13 & Lagoonal \\
\hline 14 & Limestone \\
\hline 15 & Marl \\
\hline 16 & Metamorphic \\
\hline 17 & Paleozoic sediments \\
\hline 18 & Sedimentary \\
\hline 20 & Tertiary basalts \\
\hline & \\
\hline & Volcanic \\
\hline
\end{tabular}

Table S3. Primary Land Use Categories

\begin{tabular}{|c|c|c|c|}
\hline $\begin{array}{l}\text { Land } \\
\text { Use } \\
\text { Code }\end{array}$ & Description & Details & $\begin{array}{l}\text { Category } \\
\text { for Land } \\
\text { Use Proxy }\end{array}$ \\
\hline 1 & Residential & Housing & Urbanized \\
\hline 2 & Commercial & $\begin{array}{l}\text { Shops, restaurants, cinemas, amusement parks, bars, } \\
\text { hospitals, offices, parking lots }\end{array}$ & Urbanized \\
\hline 3 & Industrial & $\begin{array}{l}\text { Manufacturing, warehouses, noxious/dangerous } \\
\text { production (e.g. tannery, oil refinery) }\end{array}$ & Urbanized \\
\hline 4 & Extractive Industries & Mining (sand, rock, metals, salt, water, dredging) & Urbanized \\
\hline 5 & Primary Production & $\begin{array}{l}\text { Native, grazing, ag, farming, orchards, forestry, } \\
\text { aquaculture }\end{array}$ & Agricultura \\
\hline 6 & $\begin{array}{l}\text { Infrastructure and } \\
\text { utilities }\end{array}$ & $\begin{array}{l}\text { Gas, electricity, waste, recycling, water, roads, railways, } \\
\text { tramways, wharfs, post }\end{array}$ & Urbanized \\
\hline 7 & Community services & $\begin{array}{l}\text { Schools, day-care, hospitals, police, courts, emergency } \\
\text { services, prisons, churches, sporting halls, government } \\
\text { buildings and bases }\end{array}$ & Urbanized \\
\hline 8 & $\begin{array}{l}\text { Sport Heritage and } \\
\text { Culture }\end{array}$ & $\begin{array}{l}\text { Sports complexes, race tracks, fields, libraries, museums, } \\
\text { botanical gardens, aquariums, memorials }\end{array}$ & Urbanized \\
\hline 9 & $\begin{array}{l}\text { National parks, } \\
\text { conservation areas, } \\
\text { forest reserves, and } \\
\text { natural water reserves }\end{array}$ & Reserves, wetlands, national parks, protected areas & Natural \\
\hline
\end{tabular}


Table S4. Covariates included in the twelve global models for 30-cm deep sediment blue C stocks. To avoid correlation between covariates, each model contained six fixed covariates and site as a random effect. $\mathrm{EVC}=$ ecological vegetation class (for tidal marsh); $\mathrm{D}=$ distance; $\mathrm{FW}=$ freshwater channel; population = mean population; $\%=$ proportion of the catchment area of the named land use.

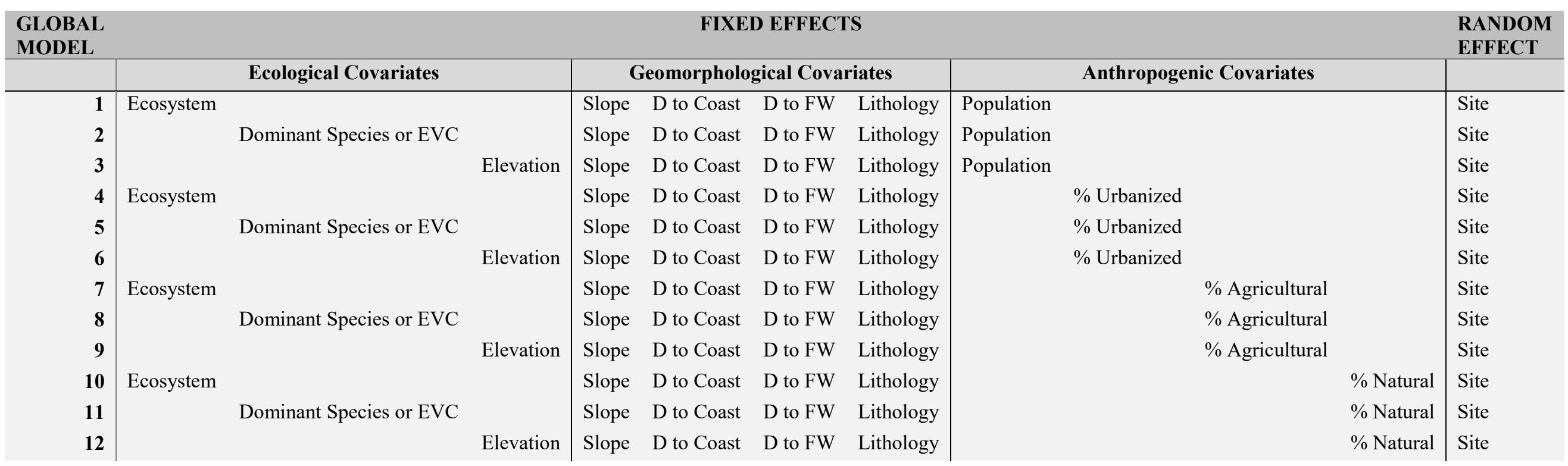


Table S5. $\mathrm{AIC}_{C}$ ranking of the twelve global models for $30-\mathrm{cm}$ deep sediment blue $\mathrm{C}$ stocks.

\begin{tabular}{lllllll}
\hline Global Model & K & AIC $\boldsymbol{C}$ & Delta_AIC $\boldsymbol{C}$ & AICCWt $_{\boldsymbol{C}}$ & Cum.Wt & LL \\
\hline 11 & 30 & 1823.61 & 0.00 & 0.70 & 0.70 & -876.27 \\
5 & 30 & 1826.39 & 2.79 & 0.17 & 0.87 & -877.66 \\
2 & 30 & 1828.21 & 4.60 & 0.07 & 0.94 & -878.57 \\
8 & 30 & 1828.65 & 5.04 & 0.06 & 1.00 & -878.79 \\
10 & 24 & 1858.48 & 34.87 & 0.00 & 1.00 & -876.27 \\
4 & 24 & 1860.99 & 37.38 & 0.00 & 1.00 & -903.05 \\
1 & 24 & 1864.31 & 40.71 & 0.00 & 1.00 & -904.71 \\
7 & 24 & 1864.75 & 41.14 & 0.00 & 1.00 & -904.93 \\
12 & 23 & 1886.26 & 62.65 & 0.00 & 1.00 & -916.98 \\
6 & 23 & 1893.33 & 69.72 & 0.00 & 1.00 & -920.51 \\
9 & 23 & 1895.62 & 72.01 & 0.00 & 1.00 & -921.66 \\
3 & 23 & 1895.97 & 72.37 & 0.00 & 1.00 & -921.83 \\
\hline
\end{tabular}


Table S6. Dredge products of dominant species/EVC global models. Top models resulting from dredging the global models (based on delta AIC $<2$ ) were used to produce averaged models and parameter estimates. Note the anthropogenic covariate distinguishes the global models from one another. $\mathrm{EVC}=\mathrm{ecological}$ vegetation class; $N / A=$ parameter was not included in the dredge product model; "+” = factor included in model.

\begin{tabular}{|c|c|c|c|c|c|c|c|c|c|c|c|c|c|}
\hline Global & Dredge & & Distance to & Dominant & Primary & Distance to & & Proportion & & & & & \\
\hline Model & product & Intercept & coast & species/EVC & lithology & freshwater & Slope & natural & df & logLik & AICc & delta & weight \\
\hline \multirow[t]{8}{*}{11} & 3 & 0.0168564 & NA & + & $N A$ & NA & NA & NA & 11 & -895.369 & 1814.15 & 0 & $1.29 \mathrm{E}-01$ \\
\hline & 4 & 0.0189982 & -0.0020353 & + & NA & NA & $N A$ & NA & 12 & -894.3325 & 1814.342 & 0.1926387 & $1.17 \mathrm{E}-01$ \\
\hline & 12 & 0.018197 & -0.0022854 & + & NA & -0.0025109 & $N A$ & NA & 13 & -893.5455 & 1815.059 & 0.9088236 & $8.17 E-02$ \\
\hline & 36 & 0.0189583 & -0.0023732 & + & NA & NA & NA & 0.001781 & 13 & -893.6158 & 1815.199 & 1.0493365 & $7.61 \mathrm{E}-02$ \\
\hline & 11 & 0.0157893 & $N A$ & + & NA & -0.0019917 & $N A$ & NA & 12 & -894.8916 & 1815.461 & 1.31077 & $6.68 \mathrm{E}-02$ \\
\hline & 19 & 0.0166106 & NA & + & NA & NA & $-6.83 \mathrm{E}-04$ & NA & 12 & -894.9389 & 1815.555 & 1.4055444 & $6.37 \mathrm{E}-02$ \\
\hline & 20 & 0.0190315 & -0.0021158 & + & NA & NA & $-7.06 \mathrm{E}-04$ & NA & 13 & -893.8551 & 1815.678 & 1.5279401 & $5.99 \mathrm{E}-02$ \\
\hline & 35 & 0.0164176 & NA & + & NA & NA & NA & 0.0012525 & 12 & -895.0034 & 1815.684 & 1.534444 & 5.97E-02 \\
\hline Global & Dredge & & Distance to & Dominant & Primary & Distance to & & Proportion & & & & & \\
\hline Model & product & Intercept & coast & species/EVC & lithology & freshwater & Slope & urbanized & df & logLik & AICc & delta & weight \\
\hline \multirow[t]{12}{*}{5} & 3 & 0.01685643 & NA & + & NA & NA & NA & NA & 11 & -895.3690 & 1814.150 & 0.0000000 & $1.113112 \mathrm{e}-01$ \\
\hline & 4 & 0.01899821 & -0.0020353223 & + & NA & NA & NA & NA & 12 & -894.3325 & 1814.342 & 0.1926387 & $1.010899 \mathrm{e}-01$ \\
\hline & 36 & 0.01800898 & -0.0019729499 & + & NA & NA & NA & -0.0021066956 & 13 & -893.2183 & 1814.404 & 0.2543995 & $9.801593 \mathrm{e}-02$ \\
\hline & 35 & 0.01551172 & $N A$ & + & NA & NA & $N A$ & -0.0019051529 & 12 & -894.3987 & 1814.475 & 0.3251377 & $9.460978 \mathrm{e}-02$ \\
\hline & 12 & 0.01819697 & -0.0022853901 & + & NA & -0.002510929 & NA & NA & 13 & -893.5455 & 1815.059 & 0.9088236 & $7.066271 \mathrm{e}-02$ \\
\hline & 44 & 0.01711336 & -0.0023424427 & + & NA & -0.002368407 & $N A$ & -0.0020726030 & 14 & -892.4650 & 1815.213 & 1.0628511 & $6.542498 \mathrm{e}-02$ \\
\hline & 11 & 0.01578925 & NA & + & NA & -0.001991729 & NA & NA & 12 & -894.8916 & 1815.461 & 1.3107700 & $5.779746 \mathrm{e}-02$ \\
\hline & 19 & 0.01661063 & NA & + & NA & NA & -0.0006827148 & NA $\mid$ & 12 & -894.9389 & 1815.555 & 1.4055444 & $5.512248 \mathrm{e}-02$ \\
\hline & 20 & 0.01903147 & -0.0021157993 & + & NA & NA & -0.0007056025 & NA & 13 & -893.8551 & 1815.678 & 1.5279401 & $5.185025 \mathrm{e}-02$ \\
\hline & 43 & 0.01470687 & $N A$ & + & NA & -0.002029556 & $N A$ & -0.0019050897 & 13 & -893.9474 & 1815.862 & 1.7126661 & $4.727571 \mathrm{e}-02$ \\
\hline & 52 & 0.01798426 & -0.0021412230 & + & NA & NA & -0.0006979693 & -0.0020573538 & 14 & -892.7948 & 1815.872 & 1.7225099 & $4.704360 \mathrm{e}-02$ \\
\hline & 51 & 0.01478040 & NA & + & NA & NA & -0.0005631329 & -0.0021105856 & 13 & -894.0431 & 1816.054 & 1.9040556 & $4.296140 \mathrm{e}-02$ \\
\hline Global & Dredge & & Distance to & Dominant & Primary & Distance to & & $\begin{array}{r}\text { Mean } \\
\text { Population }\end{array}$ & & & & & \\
\hline Model & product & Intercept & coast & species/EVC & lithology & freshwater & Slope & Density & df & logLik & AICc & delta & weight \\
\hline \multirow[t]{6}{*}{2} & 3 & 0.01685643 & NA & + & NA & NA & NA & NA & 11 & -895.3690 & 1814.150 & 0.0000000 & $1.543957 \mathrm{e}-01$ \\
\hline & 4 & 0.01899821 & -0.0020353223 & + & NA & NA & NA & NA & 12 & -894.3325 & 1814.342 & 0.1926387 & $1.402182 \mathrm{e}-01$ \\
\hline & 12 & 0.01819697 & -0.0022853901 & + & NA & -0.002510929 & $N A$ & NA & 13 & -893.5455 & 1815.059 & 0.9088236 & $9.801370 \mathrm{e}-02$ \\
\hline & 11 & 0.01578925 & NA & + & NA & -0.001991729 & NA & NA & 12 & -894.8916 & 1815.461 & 1.3107700 & $8.016877 \mathrm{e}-02$ \\
\hline & 35 & 0.01661063 & NA & + & NA & NA & -0.0006827148 & NA & 12 & -894.9389 & 1815.555 & 1.4055444 & 7.645840e-02 \\
\hline & 36 & 0.01903147 & -0.0021157993 & + & NA & NA & -0.0007056025 & NA & 13 & -893.8551 & 1815.678 & 1.5279401 & $7.191961 \mathrm{e}-02$ \\
\hline $\begin{array}{l}\text { Global } \\
\text { Model }\end{array}$ & $\begin{array}{l}\text { Dredge } \\
\text { product }\end{array}$ & Intercept & $\begin{array}{r}\text { Distance to } \\
\text { coast } \\
\end{array}$ & $\begin{array}{c}\text { Dominant } \\
\text { species/EVC }\end{array}$ & $\begin{array}{l}\text { Primary } \\
\text { lithology }\end{array}$ & $\begin{array}{l}\text { Distance to } \\
\text { freshwater }\end{array}$ & Slope & $\begin{array}{l}\text { Proportion } \\
\text { agricultural }\end{array}$ & df & logLik & AICc & delta & weight \\
\hline \multirow[t]{3}{*}{8} & 3 & 0.01685643 & NA & + & NA & $N A$ & NA & $N A$ & 11 & -895.3690 & 1814.150 & 0.0000000 & $1.581714 \mathrm{e}-01$ \\
\hline & 4 & 0.01899821 & -0.0020353223 & + & NA & NA & $N A$ & NA & 12 & -894.3325 & 1814.342 & 0.1926387 & $1.436472 \mathrm{e}-01$ \\
\hline & 12 & 0.01819697 & -0.0022853901 & + & NA & -0.002510929 & $N A$ & NA & 13 & -893.5455 & 1815.059 & 0.9088236 & $1.004106 \mathrm{e}-01$ \\
\hline
\end{tabular}


Table S7. Full output table of averaged model parameter estimates containing dominant species/ecological vegetation class $(\mathrm{EVC})$ as the ecological variable.

\begin{tabular}{|c|c|c|c|c|c|c|}
\hline \multicolumn{7}{|l|}{ Averaged model 11} \\
\hline \multirow[b]{2}{*}{ Parameter } & \multirow[b]{2}{*}{ Estimate } & \multirow[b]{2}{*}{$\begin{array}{l}\text { Adjusted } \\
\text { SE }\end{array}$} & \multicolumn{2}{|c|}{$\begin{array}{c}\text { Confidence } \\
\text { interval }\end{array}$} & \multirow{2}{*}{$\begin{array}{l}\text { Relative } \\
\text { importance }\end{array}$} & \multirow{2}{*}{$\begin{array}{l}\mathrm{N} \\
\text { containing } \\
\text { models }\end{array}$} \\
\hline & & & $2.5 \%$ & $97.5 \%$ & & \\
\hline $\begin{array}{l}\text { Intercept: } \\
\text { Coastal tussock saltmarsh }\end{array}$ & 0.0177 & 0.0043 & 0.0093 & 0.0260 & & \\
\hline $\begin{array}{l}\text { Factor (dominant sp/EVC): } \\
\text { Wet saltmarsh herbland }\end{array}$ & 0.0012 & 0.0041 & -0.0068 & 0.0092 & 1.00 & 8 \\
\hline $\begin{array}{l}\text { Factor (dominant sp/EVC): } \\
\text { wet saltmarsh shrubland }\end{array}$ & -0.0027 & 0.0042 & -0.0110 & 0.0056 & $"$ & $"$ \\
\hline $\begin{array}{l}\text { Factor (dominant } \mathrm{sp} / \mathrm{EVC} \text { ): } \\
\text { A. marina mangroves }\end{array}$ & 0.0011 & 0.0041 & -0.0070 & 0.0092 & $"$ & $"$ \\
\hline $\begin{array}{l}\text { Factor (dominant } \mathrm{sp} / \mathrm{EVC} \text { ): } \\
\text { L. marina seagrass }\end{array}$ & -0.0024 & 0.0051 & -0.0123 & 0.0075 & $"$ & $"$ \\
\hline $\begin{array}{l}\text { Factor (dominant } \mathrm{sp} / \mathrm{EVC} \text { ): } \\
\text { P. australis } \text { seagrass }\end{array}$ & 0.0394 & 0.0179 & 0.0043 & 0.0745 & $"$ & $"$ \\
\hline $\begin{array}{l}\text { Factor (dominant } \mathrm{sp} / \mathrm{EVC} \text { ): } \\
\text { R. megacarpa seagrass }\end{array}$ & 0.0903 & 0.0313 & 0.0289 & 0.1518 & $"$ & " \\
\hline $\begin{array}{l}\text { Factor (dominant } \mathrm{sp} / \mathrm{EVC} \text { ): } \\
\text { Z. muelleri seagrass }\end{array}$ & 0.0291 & 0.0047 & 0.0198 & 0.0384 & $"$ & $"$ \\
\hline $\begin{array}{l}\text { Factor (dominant } \mathrm{sp} / \mathrm{EVC} \text { ): } \\
\text { Z. nigricaulis seagrass }\end{array}$ & 0.0397 & 0.0172 & 0.0060 & 0.0735 & $"$ & " \\
\hline Distance to coast & -0.0011 & 0.0015 & -0.0041 & 0.0019 & 0.51 & 4 \\
\hline Distance to freshwater & -0.0005 & 0.0014 & -0.0032 & 0.0022 & 0.23 & 2 \\
\hline Proportion natural & 0.0003 & 0.0009 & -0.0015 & 0.0022 & 0.21 & 2 \\
\hline Slope & -0.0001 & 0.0004 & -0.0015 & 0.0022 & 0.19 & 2 \\
\hline
\end{tabular}

\begin{tabular}{|c|c|c|c|c|c|c|}
\hline \multicolumn{7}{|l|}{ Averaged model 5} \\
\hline \multirow[b]{2}{*}{ Parameter } & \multirow[b]{2}{*}{ Estimate } & \multirow[b]{2}{*}{$\begin{array}{l}\text { Adjusted } \\
\text { SE }\end{array}$} & \multicolumn{2}{|c|}{$\begin{array}{c}\text { Confidence } \\
\text { interval }\end{array}$} & \multirow{2}{*}{$\begin{array}{l}\text { Relative } \\
\text { importance }\end{array}$} & \multirow{2}{*}{$\begin{array}{l}\mathrm{N} \\
\text { containing } \\
\text { models }\end{array}$} \\
\hline & & & $2.5 \%$ & $97.5 \%$ & & \\
\hline $\begin{array}{l}\text { Intercept: } \\
\text { Coastal tussock saltmarsh }\end{array}$ & 0.0171 & 0.0042 & 0.0088 & 0.0254 & & \\
\hline $\begin{array}{l}\text { Factor (dominant sp/EVC): } \\
\text { Wet saltmarsh herbland }\end{array}$ & 0.0013 & 0.0040 & -0.0066 & 0.0092 & 1.00 & 12 \\
\hline $\begin{array}{l}\text { Factor (dominant } \mathrm{sp} / \mathrm{EVC} \text { ): } \\
\text { wet saltmarsh shrubland }\end{array}$ & -0.0023 & 0.0042 & -0.0107 & 0.0060 & $"$ & $"$ \\
\hline $\begin{array}{l}\text { Factor (dominant } \mathrm{sp} / \mathrm{EVC}) \text { : } \\
\text { A. marina mangroves }\end{array}$ & 0.0015 & 0.0041 & -0.0066 & 0.0095 & $"$ & $"$ \\
\hline $\begin{array}{l}\text { Factor (dominant } \mathrm{sp} / \mathrm{EVC} \text { ): } \\
\text { L. marina seagrass }\end{array}$ & -0.0020 & 0.0051 & -0.0119 & 0.0080 & $"$ & $"$ \\
\hline $\begin{array}{l}\text { Factor (dominant } \mathrm{sp} / \mathrm{EVC}) \text { : } \\
\text { P. australis seagrass }\end{array}$ & 0.0405 & 0.0179 & 0.0054 & 0.0756 & $"$ & $"$ \\
\hline $\begin{array}{l}\text { Factor (dominant } \mathrm{sp} / \mathrm{EVC} \text { ): } \\
\text { R. megacarpa seagrass }\end{array}$ & 0.0908 & 0.0314 & 0.0293 & 0.1523 & $"$ & $"$ \\
\hline $\begin{array}{l}\text { Factor (dominant } \mathrm{sp} / \mathrm{EVC} \text { ): } \\
\text { Z. muelleri seagrass }\end{array}$ & 0.0295 & 0.0047 & 0.0202 & 0.0388 & $"$ & $"$ \\
\hline $\begin{array}{l}\text { Factor (dominant } \mathrm{sp} / \mathrm{EVC} \text { ): } \\
\text { Z. nigricaulis seagrass }\end{array}$ & 0.0389 & 0.0172 & 0.0052 & 0.0727 & $"$ & $"$ \\
\hline Distance to coast & -0.0011 & 0.0015 & -0.0040 & 0.0018 & 0.51 & 6 \\
\hline
\end{tabular}




\begin{tabular}{|c|c|c|c|c|c|c|}
\hline Proportion Urbanized & -0.0010 & 0.0014 & -0.0037 & 0.0018 & 0.47 & 6 \\
\hline Distance to freshwater & -0.0006 & 0.0015 & -0.0036 & 0.0023 & 0.29 & 4 \\
\hline Slope & -0.0002 & 0.0005 & -0.0010 & 0.0007 & 0.23 & 4 \\
\hline \multicolumn{7}{|l|}{ Averaged model 2} \\
\hline \multirow[b]{2}{*}{ Parameter } & \multirow[b]{2}{*}{ Estimate } & \multirow[b]{2}{*}{$\begin{array}{l}\text { Adjusted } \\
\text { SE }\end{array}$} & \multicolumn{2}{|c|}{$\begin{array}{c}\text { Confidence } \\
\text { interval }\end{array}$} & \multirow{2}{*}{$\begin{array}{l}\text { Relative } \\
\text { importance }\end{array}$} & \multirow{2}{*}{$\begin{array}{l}\mathrm{N} \\
\text { containing } \\
\text { models }\end{array}$} \\
\hline & & & $2.5 \%$ & $97.5 \%$ & & \\
\hline $\begin{array}{l}\text { Intercept: } \\
\text { Coastal tussock saltmarsh }\end{array}$ & 0.0176 & 0.0042 & 0.0093 & 0.0260 & & \\
\hline $\begin{array}{l}\text { Factor (dominant sp/EVC): } \\
\text { Wet saltmarsh herbland }\end{array}$ & 0.0011 & 0.0041 & -0.0069 & 0.0091 & 1.00 & 6 \\
\hline $\begin{array}{l}\text { Factor (dominant sp/EVC): } \\
\text { wet saltmarsh shrubland }\end{array}$ & -0.0028 & 0.0042 & -0.0111 & 0.0055 & $"$ & $"$ \\
\hline $\begin{array}{l}\text { Factor (dominant } \mathrm{sp} / \mathrm{EVC}) \text { : } \\
\text { A. marina mangroves }\end{array}$ & 0.0011 & 0.0041 & -0.0070 & 0.0092 & $"$ & " \\
\hline $\begin{array}{l}\text { Factor (dominant } \mathrm{sp} / \mathrm{EVC} \text { ): } \\
\text { L. marina seagrass }\end{array}$ & -0.0024 & 0.0051 & -0.0124 & 0.0075 & $"$ & " \\
\hline $\begin{array}{l}\text { Factor (dominant } \mathrm{sp} / \mathrm{EVC} \text { ): } \\
\text { P. australis seagrass }\end{array}$ & 0.0412 & 0.0180 & 0.0058 & 0.0765 & $"$ & " \\
\hline $\begin{array}{l}\text { Factor (dominant } \mathrm{sp} / \mathrm{EVC} \text { ): } \\
R \text {. megacarpa seagrass }\end{array}$ & 0.0909 & 0.0313 & 0.0296 & 0.1522 & $"$ & " \\
\hline $\begin{array}{l}\text { Factor (dominant } \mathrm{sp} / \mathrm{EVC} \text { ): } \\
\text { Z. muelleri seagrass }\end{array}$ & 0.0292 & 0.0047 & 0.0199 & 0.0385 & $"$ & " \\
\hline $\begin{array}{l}\text { Factor (dominant } \mathrm{sp} / \mathrm{EVC} \text { ): } \\
\text { Z. nigricaulis seagrass }\end{array}$ & 0.0398 & 0.0172 & 0.0060 & 0.0736 & $"$ & " \\
\hline Distance to coast & -0.0011 & 0.0015 & -0.0040 & 0.0019 & 0.50 & 3 \\
\hline Distance to freshwater & -0.0007 & 0.0015 & -0.0036 & 0.0023 & 0.29 & 2 \\
\hline Slope & -0.0002 & 0.0005 & -0.0011 & 0.0007 & 0.24 & 2 \\
\hline
\end{tabular}


Table S8. Dredge products of ecosystem global models. Top models resulting from dredging the global models (based on delta AIC $C<2)$ were used to produce averaged models and parameter estimates. Note the anthropogenic covariate distinguishes the global models from one another. $N / A=$ parameter was not included in the dredge product model; "+" = factor included in model.

\begin{tabular}{|c|c|c|c|c|c|c|c|c|c|c|c|c|c|}
\hline $\begin{array}{l}\text { Global } \\
\text { Model }\end{array}$ & $\begin{array}{l}\text { Dredge } \\
\text { product }\end{array}$ & Intercept & $\begin{array}{l}\text { Distance } \\
\text { to coast }\end{array}$ & Ecosystem & $\begin{array}{r}\text { Primary } \\
\text { lithology }\end{array}$ & $\begin{array}{l}\text { Distance to } \\
\text { freshwater }\end{array}$ & Slope & $\begin{array}{r}\text { Proportion } \\
\text { natural }\end{array}$ & df & logLik & AICc & delta & weight \\
\hline \multirow[t]{5}{*}{10} & 36 & 0.0181 & -0.0020 & + & NA & NA & NA & 0.0029 & 7 & -916.37 & 1847.33 & 0.00 & 0.1583 \\
\hline & 35 & 0.0177 & NA & + & NA & NA & NA & 0.0023 & 6 & -917.61 & 1847.66 & 0.32 & 0.1346 \\
\hline & 3 & 0.0174 & NA & + & NA & NA & NA & NA & 5 & -918.96 & 1848.23 & 0.90 & 0.1007 \\
\hline & 52 & 0.0180 & -0.0021 & + & NA & NA & -0.0009 & 0.0030 & 8 & -915.79 & 1848.34 & 1.01 & 0.0953 \\
\hline & 51 & 0.0177 & NA & + & NA & NA & -0.0008 & 0.0023 & 7 & -917.15 & 1848.88 & 1.55 & 0.0730 \\
\hline $\begin{array}{l}\text { Global } \\
\text { Model }\end{array}$ & $\begin{array}{c}\text { Dredge } \\
\text { product }\end{array}$ & Intercept & $\begin{array}{l}\text { Distance } \\
\text { to coast }\end{array}$ & Ecosystem & $\begin{array}{l}\text { Primary } \\
\text { lithology }\end{array}$ & $\begin{array}{l}\text { Distance to } \\
\text { freshwater }\end{array}$ & Slope & $\begin{array}{r}\text { Proportion } \\
\text { urbanized }\end{array}$ & df & logLik & AICc & delta & weight \\
\hline \multirow[t]{5}{*}{4} & 35 & 0.0165 & $N A$ & + & NA & NA & $N A$ & -0.0027 & 6 & -916.94 & 1846.31 & 0.00 & 0.2036 \\
\hline & 36 & 0.0166 & -0.0014 & + & NA & NA & NA & -0.0029 & 7 & -916.09 & 1846.77 & 0.46 & 0.1615 \\
\hline & 51 & 0.0165 & NA & + & NA & NA & -0.0006 & -0.0026 & 7 & -916.60 & 1847.79 & 1.48 & 0.0969 \\
\hline & 52 & 0.0165 & -0.0015 & + & NA & NA & -0.0007 & -0.0029 & 8 & -915.70 & 1848.15 & 1.84 & 0.0810 \\
\hline & 3 & 0.0174 & NA & + & NA & NA & NA & NA & 5 & -918.96 & 1848.23 & 1.93 & 0.0778 \\
\hline $\begin{array}{l}\text { Global } \\
\text { Model }\end{array}$ & $\begin{array}{l}\text { Dredge } \\
\text { product }\end{array}$ & Intercept & $\begin{array}{c}\text { Distance } \\
\text { to coast }\end{array}$ & Ecosystem & $\begin{array}{r}\text { Primary } \\
\text { lithology }\end{array}$ & $\begin{array}{l}\text { Distance to } \\
\text { freshwater }\end{array}$ & Slope & $\begin{array}{r}\text { Mean } \\
\text { Population } \\
\text { Density }\end{array}$ & df & logLik & AICc & delta & weight \\
\hline \multirow[t]{4}{*}{1} & 3 & 0.0174 & $N A$ & + & NA & NA & NA & $N A$ & 5 & -918.96 & 1848.23 & 0.00 & 0.2036 \\
\hline & 4 & 0.0175 & -0.0012 & + & $N A$ & NA & NA & NA & 6 & -918.45 & 1849.35 & 1.11 & 0.1168 \\
\hline & 35 & 0.0173 & NA & + & NA & NA & -0.0008 & NA & 6 & -918.50 & 1849.44 & 1.21 & 0.1113 \\
\hline & 19 & 0.0172 & NA & + & NA & NA & NA & -0.0005 & 6 & -918.67 & 1849.78 & 1.54 & 0.0943 \\
\hline $\begin{array}{l}\text { Global } \\
\text { Model }\end{array}$ & $\begin{array}{l}\text { Dredge } \\
\text { product }\end{array}$ & Intercept & $\begin{array}{c}\text { Distance } \\
\text { to coast }\end{array}$ & Ecosystem & $\begin{array}{r}\text { Primary } \\
\text { lithology }\end{array}$ & $\begin{array}{l}\text { Distance to } \\
\text { freshwater }\end{array}$ & Slope & $\begin{array}{l}\text { Proportion } \\
\text { agricultural }\end{array}$ & df & logLik & AICc & delta & weight \\
\hline \multirow[t]{3}{*}{7} & 3 & 0.0174 & NA & + & NA & NA & NA & $N A$ & 5 & -918.96 & 1848.23 & 0.00 & 0.2181 \\
\hline & 4 & 0.0175 & -0.0012 & + & NA & NA & NA & NA & 6 & -918.45 & 1849.35 & 1.11 & 0.1251 \\
\hline & 19 & 0.0173 & NA & + & NA & NA & -0.0008 & NA & 6 & -918.50 & 1849.44 & 1.21 & 0.1192 \\
\hline
\end{tabular}


Table S9. Full output table of averaged model parameter estimates containing ecosystem as the ecological variable.

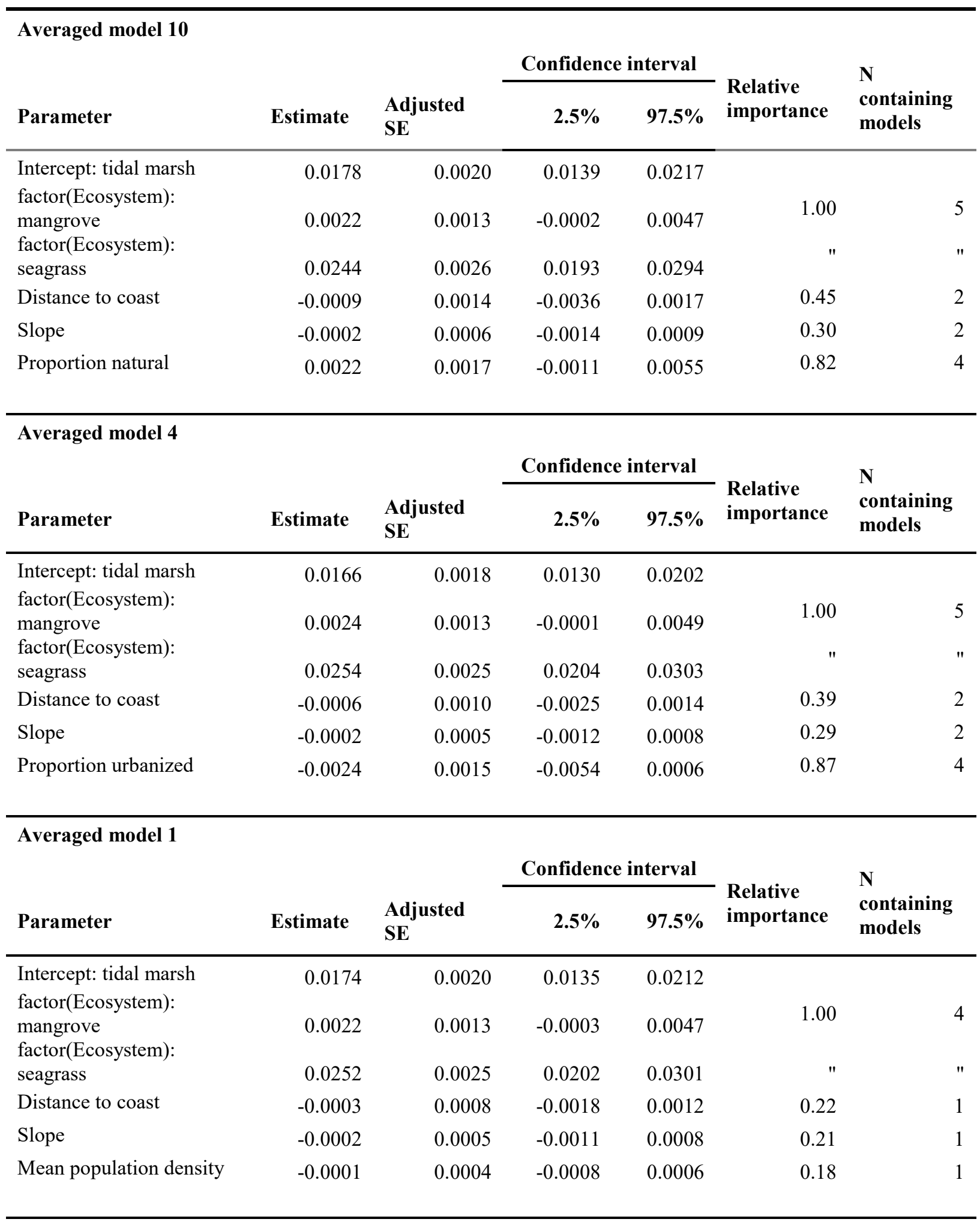




\begin{tabular}{|c|c|c|c|c|c|c|}
\hline \multicolumn{7}{|l|}{ Averaged model 7} \\
\hline \multirow[b]{2}{*}{ Parameter } & \multirow[b]{2}{*}{ Estimate } & \multirow[b]{2}{*}{$\begin{array}{l}\text { Adjusted } \\
\text { SE }\end{array}$} & \multicolumn{2}{|c|}{ Confidence interval } & \multirow{2}{*}{$\begin{array}{l}\text { Relative } \\
\text { importance }\end{array}$} & \multirow{2}{*}{$\begin{array}{l}\mathbf{N} \\
\text { containing } \\
\text { models }\end{array}$} \\
\hline & & & $2.5 \%$ & $97.5 \%$ & & \\
\hline \multirow{3}{*}{$\begin{array}{l}\text { Intercept (tidal marsh) } \\
\text { factor(Ecosystem): } \\
\text { mangrove } \\
\text { factor(Ecosystem): } \\
\text { seagrass }\end{array}$} & 0.0174 & 0.0020 & 0.0136 & 0.0213 & \multirow[b]{2}{*}{1.00} & \multirow[b]{2}{*}{3} \\
\hline & 0.0022 & 0.0013 & -0.0003 & 0.0047 & & \\
\hline & 0.0252 & 0.0025 & 0.0202 & 0.0301 & $"$ & " \\
\hline Distance to coast & -0.0003 & 0.0008 & -0.0020 & 0.0013 & 0.27 & 1 \\
\hline Slope & -0.0002 & 0.0005 & -0.0012 & 0.0008 & 0.26 & 1 \\
\hline
\end{tabular}


Table S10. Data availability

\begin{tabular}{|c|c|c|}
\hline Data Item & Description & Data Source \& Location \\
\hline $\begin{array}{l}\text { Carbon Stock } \\
\text { Dataset }\end{array}$ & $\begin{array}{l}\text { Percent organic carbon and dry bulk density } \\
\text { data for sediment sampled to } 30 \mathrm{~cm} \text { deep in } \\
96 \text { blue carbon ecosystems (saltmarshes, } \\
\text { mangrove forests, and seagrass meadows) } \\
\text { across Victoria, Australia. }\end{array}$ & $\begin{array}{l}\text { Ewers Lewis et al. } 2018 \text { Ecosystems; } \\
\text { Dataset available on Harvard Dataverse, } \\
\text { https://doi.org/10.7910/DVN/6PFBO0 }\end{array}$ \\
\hline $\begin{array}{l}\text { Ecosystem } \\
\text { Extent Vectors }\end{array}$ & $\begin{array}{l}\text { 1. Mangrove areal extent in Victoria, } \\
\text { Australia; saltmarsh areal extent and } \\
\text { ecological vegetation classes in Victoria, } \\
\text { Australia. } \\
\text { 2. Seagrass areal extent in the major bays and } \\
\text { estuaries of Victoria, Australia. } \\
\text { a. Port Phillip Bay } \\
\text { b. Western Port Bay } \\
\text { c. Corner Inlet and Nooramunga } \\
\text { d. Gippsland Lakes } \\
\text { e. Minor Inlets of Victoria }\end{array}$ & 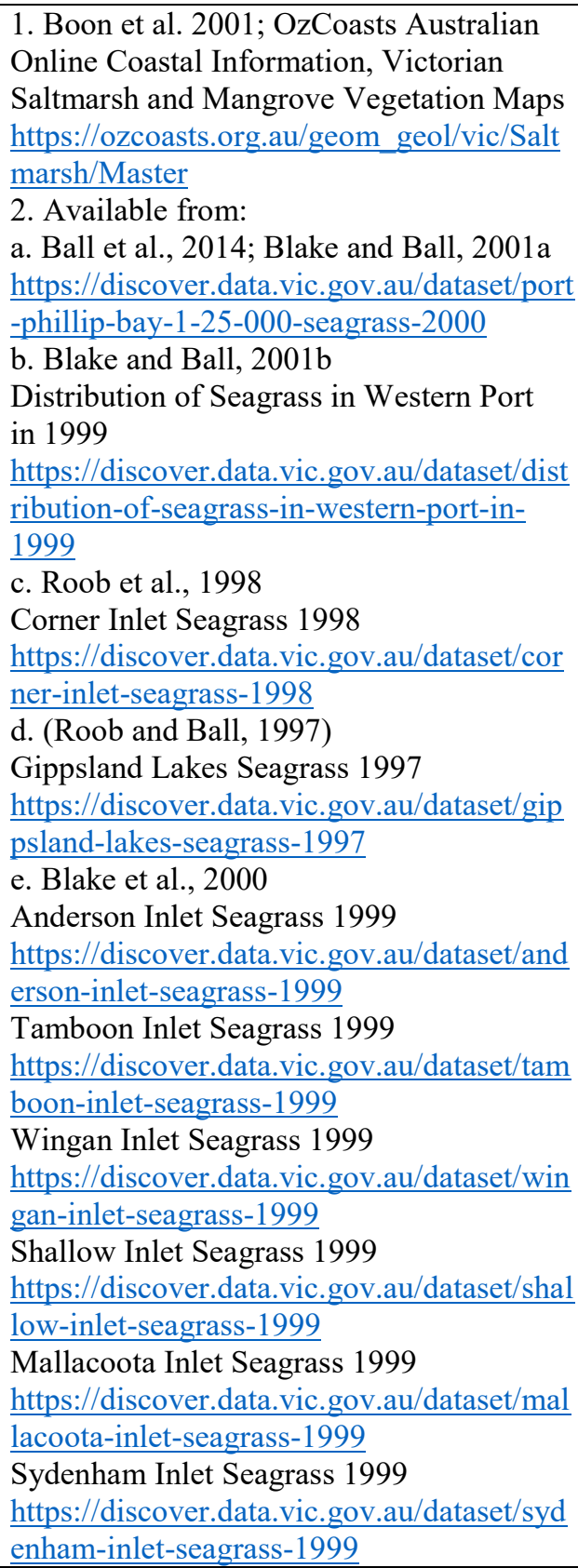 \\
\hline $\begin{array}{l}\text { Elevation } \\
\text { Raster }\end{array}$ & $\begin{array}{l}\text { A gap free digital elevation model (DEM) for } \\
\text { the coastal region of Victoria, Australia, that } \\
\text { combines } 2.5 \mathrm{~m} \text { and } 10 \mathrm{~m} \text { DEMs. }\end{array}$ & $\begin{array}{l}\text { Victorian Coastal Digital Elevation Model } \\
\text { (VCDEM 2017) } \\
\text { https://vmdp.deakin.edu.au/geonetwork/srv } \\
\text { leng/metadata.show?uuid=8d3ccf63-ee85- } \\
\text { 41cd-917e-933624a50b2e }\end{array}$ \\
\hline $\begin{array}{l}\text { Freshwater } \\
\text { Vectors }\end{array}$ & $\begin{array}{l}\text { Location of channels and other freshwater } \\
\text { objects in Victoria, Australia. }\end{array}$ & $\begin{array}{l}\text { Vicmap Hydro 1:25,000 } \\
\text { Victorian Government Data portal } \\
\text { https://discover.data.vic.gov.au/dataset/vic } \\
\text { map-hydro-1-25-000 }\end{array}$ \\
\hline
\end{tabular}




\begin{tabular}{|c|c|c|}
\hline $\begin{array}{l}\text { Coastline } \\
\text { Vector }\end{array}$ & $\begin{array}{l}\text { Line delineating the coastline of Victoria, } \\
\text { Australia. }\end{array}$ & $\begin{array}{l}\text { Victorian Coastline } 2008 \\
\text { Victorian Government Data portal } \\
\text { https://discover.data.vic.gov.au/dataset/vict } \\
\text { orian-coastline-2008 } \\
\end{array}$ \\
\hline $\begin{array}{l}\text { Lithology } \\
\text { Vectors }\end{array}$ & Rock types across Victoria, Australia. & $\begin{array}{l}\text { Geomorphology of Victoria } \\
\text { Victorian Government Data portal } \\
\text { https://discover.data.vic.gov.au/dataset/geo } \\
\text { morphology-of-victoria }\end{array}$ \\
\hline $\begin{array}{l}\text { Land Use } \\
\text { Vectors }\end{array}$ & $\begin{array}{l}\text { Primary land use designations for land } \\
\text { parcels in Victoria, Australia. }\end{array}$ & $\begin{array}{l}\text { Victorian Land Use Information System } \\
2014 / 2015 \\
\text { Victorian Government Data portal } \\
\text { https://discover.data.vic.gov.au/dataset/vict } \\
\text { orian-land-use-information-system-2014- } \\
2015\end{array}$ \\
\hline $\begin{array}{l}\text { Population } \\
\text { Raster }\end{array}$ & $\begin{array}{l}\text { Human population data for Victoria, } \\
\text { Australia. }\end{array}$ & $\begin{array}{l}\text { Australian Population Grid, } 2011 \\
\text { Australian Bureau of Statistics } \\
\text { https://www.abs.gov.au/AUSSTATS/abs@, } \\
\text {.nsf/Lookup/1270.0.55.007Main+Features1 } \\
\text { 2011?OpenDocument }\end{array}$ \\
\hline R Code & $\begin{array}{l}\mathrm{R} \text { code used to identify drivers and model } \\
\text { carbon shallow sediment carbon stocks. }\end{array}$ & $\begin{array}{l}\text { This study. } \\
\text { Dataset available on Harvard Dataverse, } \\
\text { https://doi.org/10.7910/DVN/0WKEHJ }\end{array}$ \\
\hline $\begin{array}{l}\text { Model Output } \\
\text { Raster }\end{array}$ & $\begin{array}{l}\text { Shallow sediment (to } 30 \mathrm{~cm} \text { deep) carbon } \\
\text { stock predictions in blue carbon ecosystems } \\
\text { (seagrass meadows, mangrove forests, and } \\
\text { saltmarshes) in Victoria, Australia }\end{array}$ & $\begin{array}{l}\text { This study. } \\
\text { Dataset available on Harvard Dataverse, } \\
\text { https://doi.org/10.7910/DVN/UDOAUT }\end{array}$ \\
\hline
\end{tabular}




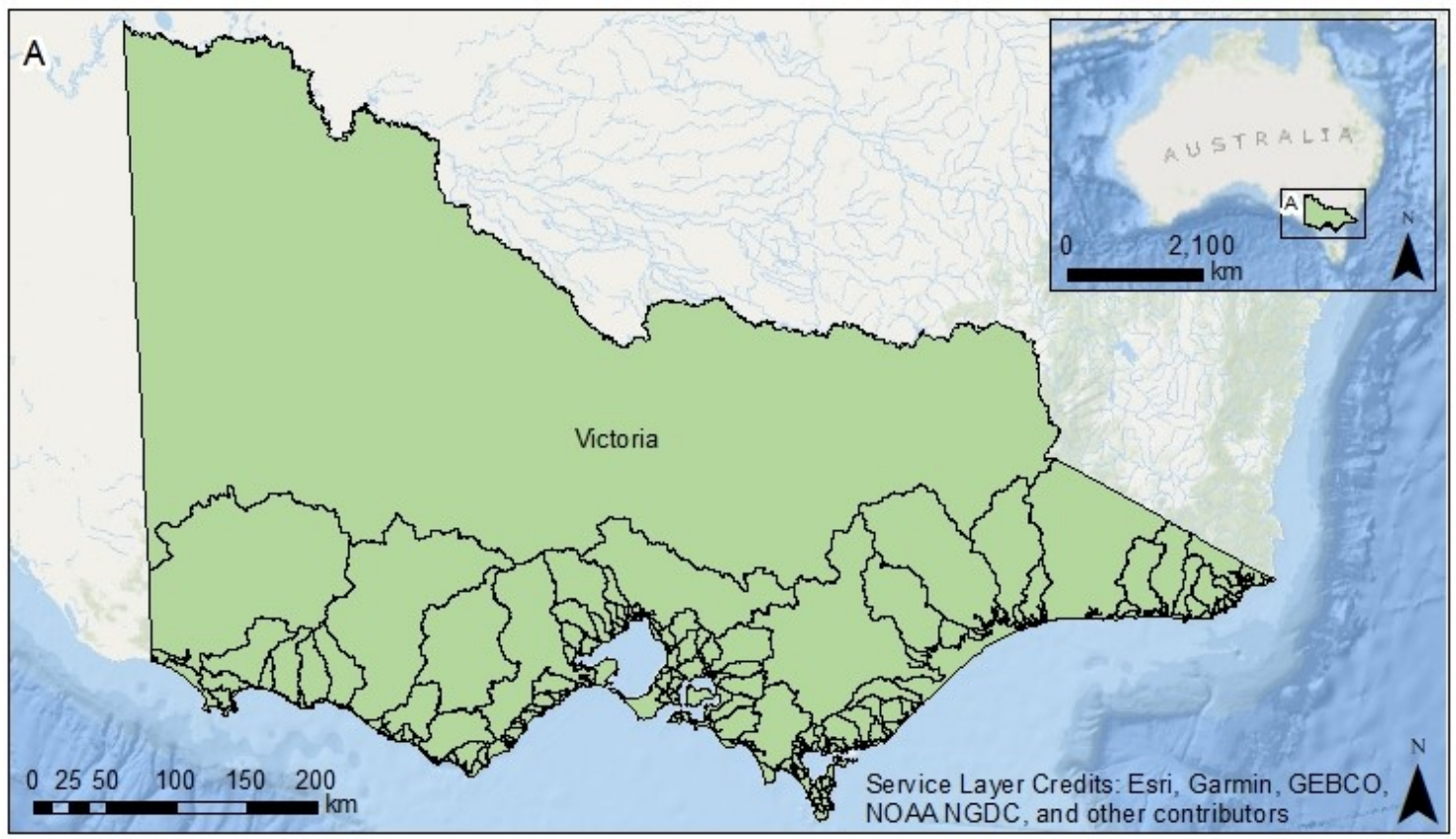

Figure S1. Fluvial and estuarine catchment regions for Victoria, Australia (Barton et al., 2008). 


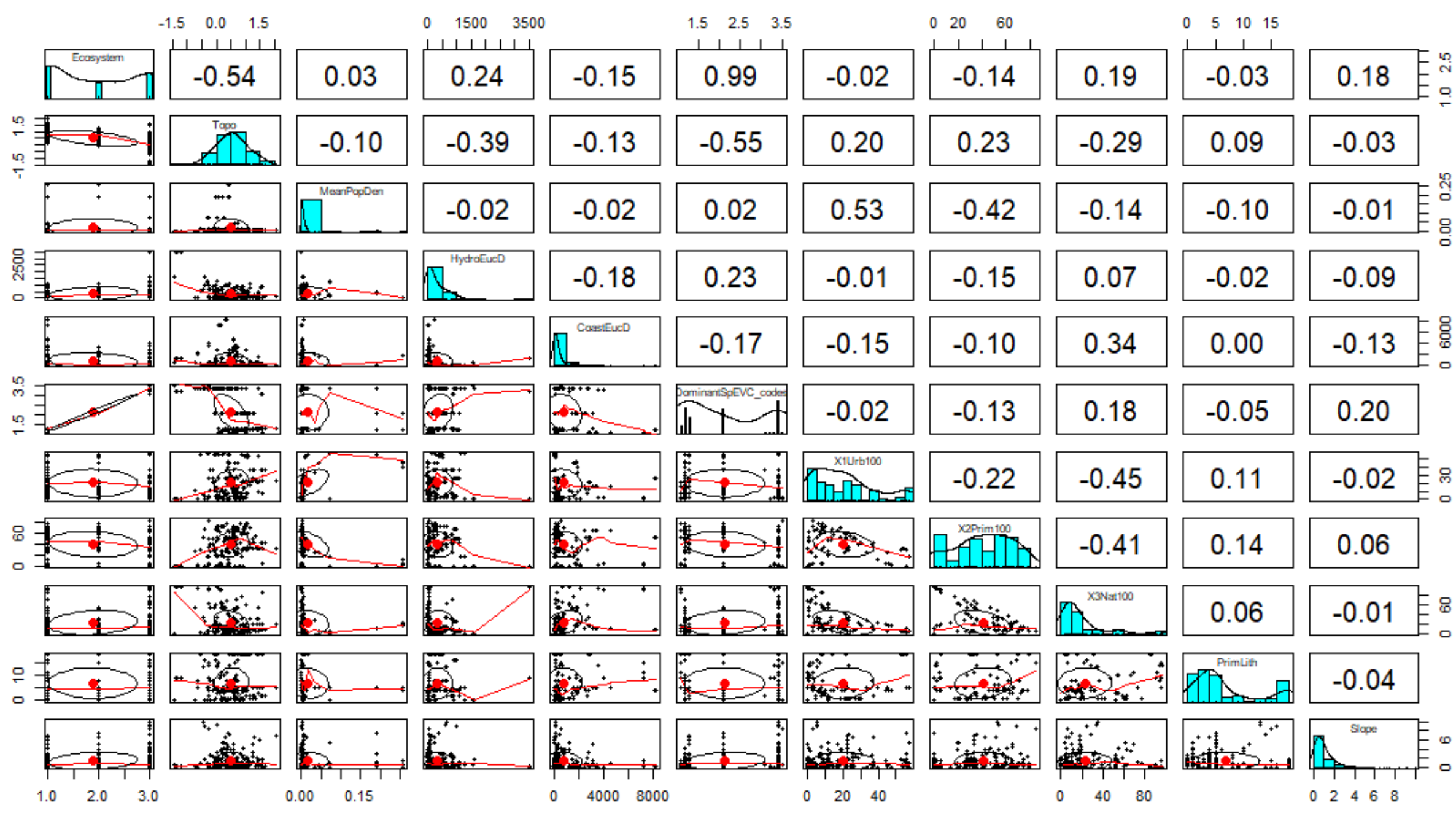

Figure S2. Pairwise plots showing correlation relationships between covariates. From top left to bottom right: ecosystem type, elevation ("topo"), mean population density ("MeanPopDen"), distance to freshwater channel ("HydroEucD"), distance to coast ("CoastEucD"), dominant species/ecological vegetation class

(“DominantSpEVC_codes"), proportion urbanized (“X1Urb100”), proportion agricultural ("X2Prim100”), proportion natural ("X3Nat100”), primary lithology (“PrimLith"), and slope. 


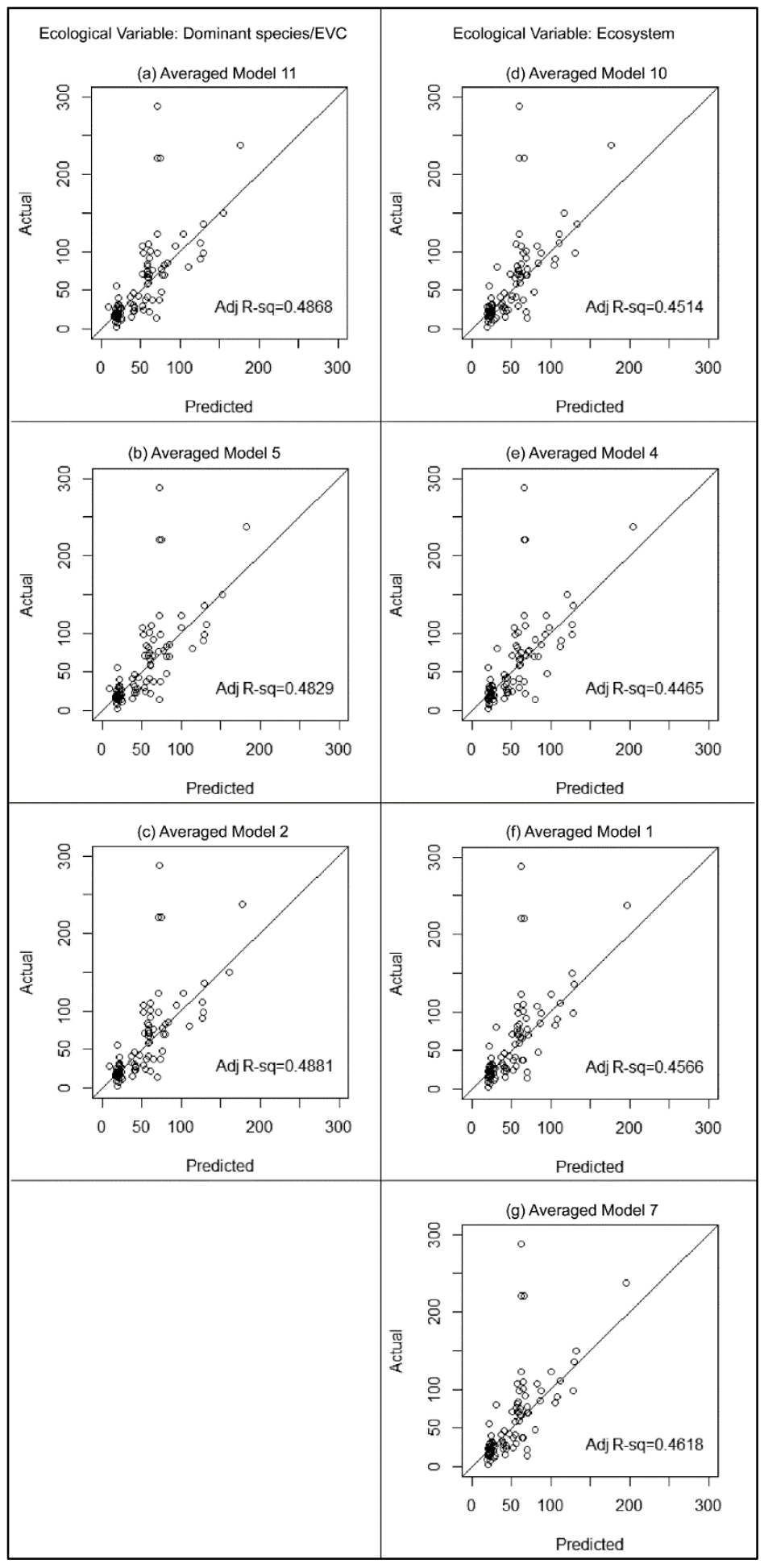

Figure S3. Predicted v. actual 30-cm deep sediment blue C stocks. Averaged model 2 produced the best predictions out of the models using dominant species/EVC as the ecological variable (left column), while averaged model 7 produced the best predictions out of the models using ecosystem as the ecological variable. Neither of the best averaged models (2 and 7) included anthropogenic covariates. Linear regressions of predicted versus actual measured $30-\mathrm{cm}$ deep sediment $\mathrm{C}$ values produced the following outputs for each averaged model: averaged model 11, residual standard error $(\mathrm{RSE})=38.36$ on 84 degrees of freedom $(\mathrm{df})$, adjusted R-squared $(\mathrm{R}$-sq(adj) $=0.4868, \mathrm{~F}$-statistic $(\mathrm{F}$-stat $)=81.63$ on 1 and $84 \mathrm{df}$, p-value $=5.044 \mathrm{e}$-14; averaged model 5, RSE $=38.51, \mathrm{R}-\mathrm{sq}(\mathrm{adj})=0.4829$, $\mathrm{F}$-stat $=80.39$ on 1 and $84 \mathrm{df}$, p-value $=6.953 \mathrm{e}-14$; averaged model 2, $\mathrm{RSE}=38.32$, R-sq(adj) $=0.4881$, $\mathrm{F}$-stat $=82.06$ on 1 and $84 \mathrm{df}$, $\mathrm{p}$-value $=4.517 \mathrm{e}-14$; averaged model 10 , $\mathrm{RSE}=39.67$, R-sq(adj) $=0.4514, \mathrm{~F}$-stat $=70.93$ on 1 and $84 \mathrm{df}$, p-value=8.645e-13; averaged model 4, RSE=39.84, $\mathrm{R}$-sq(adj) $=0.4465$, F-stat $=69.58$ on 1 and 84 df, p-value $=1.254 \mathrm{e}-12$; averaged model 1; RSE=39.48, R- 
$\mathrm{sq}(\operatorname{adj})=0.4566, \mathrm{~F}$-stat $=72.43$ on 1 and $84 \mathrm{df}, \mathrm{p}$-value $=5.73 \mathrm{e}-13$; averaged model $7, \mathrm{RSE}=39.29$, R$\mathrm{sq}(\mathrm{adj})=0.4618, \mathrm{~F}-\mathrm{stat}=73.94$ on 1 and $84 \mathrm{df}, \mathrm{p}$-value $=3.81 \mathrm{e}-13$. 